\title{
Tiling Effect Performance Analysis
}

\author{
Gwanggil Jeon \\ Department of Embedded Systems Engineering, Incheon National University \\ 119 Academy-ro, Yeonsu-gu, Incheon 406-772, Korea \\ gjeon@incheon.ac.kr
}

\begin{abstract}
In this paper, we present a tile effect generation algorithm. The presented method cuts a given pictures with small-size tiles and an original image is composed of those tiles. The size of tiles is generated by random function. We use four parameters: size of horizontal and vertical block, size of skip blocks in horizontal and vertical direction. The presented method is tested on McM dataset. Simulation results provide performance comparison in terms of PSNR, CMSE and FSIM.
\end{abstract}

Keywords: Tile effect, random function, skip block, performance assessment

\section{Introduction}

Image processing is one of signal processing shapes where input is an image [1]. In general, image processing indicates digital signal processing, where optical image processing is possible. In image processing, a filter is a tool or process which adds specific effect or removes some unwanted feature [2-4]. Image filtering is one of attributes of signal filters, where image filters have two dimensional signal while signal filters is for one dimensional signal [5-7]. By applying image filters, one may suppress or express some aspect of the images. There are different types of image filters.

The tiling effect (TE) is an approach of traditional art, which can be applied to interior decoration in an old house. A TE is often viewed in poster art. For example, some effects divide original image with thousands of small pieces to form a related much larger image. Therefore, this effect may be seen as an illusion effect.

The TE approach is classified into three categories: block-based method, pixel-based method, and image-based method. Our proposed approach is one of block-based methods. In order to apply TE approach, we uses four parameters: horizontal block size, vertical block size, horizontal skip block size, and vertical skip block size. By changing the size of four parameters, we assess the objective and visual performance. We try our best to find the most appropriate size of the block in a given image.

In this paper we propose a filter which gives tiling effect on a given image [8]. Section 2 presents a proposed filter. The proposed filter has four parameters. Section 3 describes simulation results where objective and subjective performance are compared. By changing four parameters, different effects are presented. Conclusion remarks are provided in Section 4. 


\section{Proposed Method}

\subsection{Definition of the Proposed Method}

The presented method is composed with following components. There are four parameters $\mathrm{S}_{\mathrm{HB}}, \mathrm{S}_{\mathrm{VB}}, \mathrm{S}_{\mathrm{HSB}}$, and $\mathrm{S}_{\mathrm{VSB}}$, which are defined as follows:

\section{Legend:}

$\mathrm{RF}$

random function which generates values bigger than 0 and smaller than 1 .

$\mathrm{S}_{\mathrm{HB}}$

horizontal block size

$\mathrm{S}_{\mathrm{VB}}$

vertical block size

$\mathrm{S}_{\mathrm{HSB}}$

horizontal skip block size

$\mathrm{S}_{\mathrm{VSB}}$

vertical skip block size

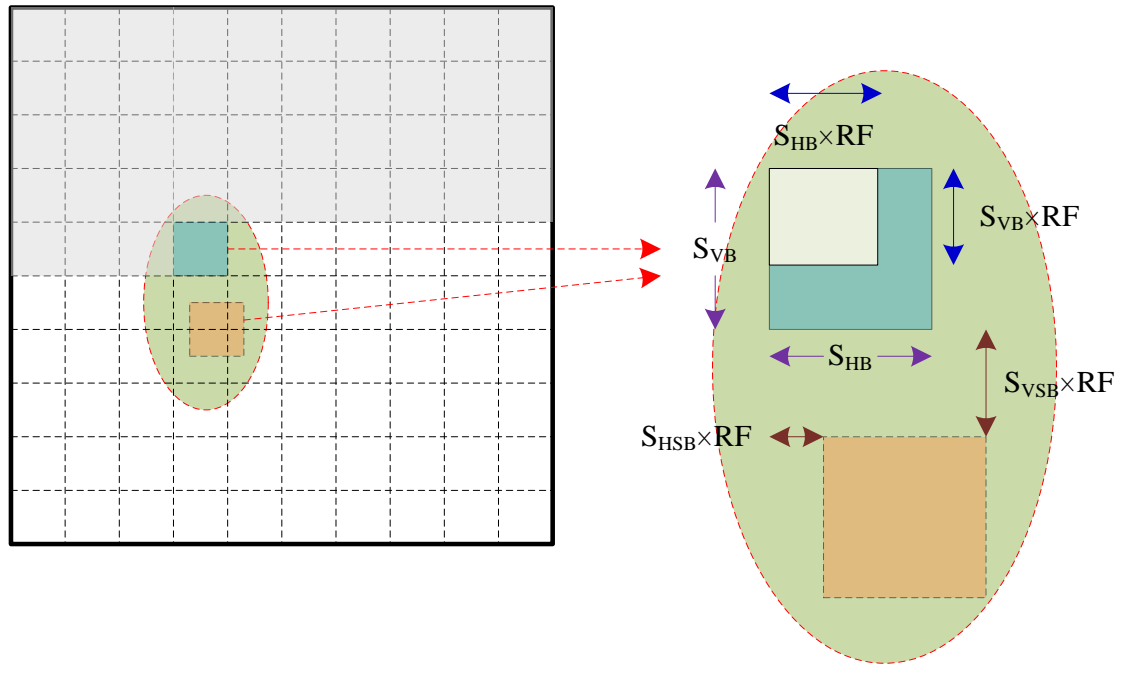

Figure 1. Concept of Four Parameters and their Corresponding Random Function

The pseudo code of the proposed method is explained as follows:

\section{Pseudo code:}

Step 1: Obtain input image.

Step 2: Determine $S_{\mathrm{HB}}$ and $S_{\mathrm{VB}}$ values.

Step 3: Determine $S_{\mathrm{HSB}}$ and $\mathrm{S}_{\mathrm{VSB}}$ values.

Step 4: Divide given image with block size, $S_{\mathrm{HB}}$ by $S_{\mathrm{VB}}$. 
Step 5: Generate random numbers [0 1].

Step 6: Generate blocks with sizes $\left[\mathrm{S}_{\mathrm{HB}} \times \mathrm{RF} \mathrm{S}_{\mathrm{VB}} \times \mathrm{RF}\right]$.

Step 7: Skip block size generation $\left[\mathrm{S}_{\mathrm{HSB}} \times \mathrm{RF} \mathrm{S}_{\mathrm{VSB}} \times \mathrm{RF}\right]$.

Step 8: Collect all generated blocks.

Step 9: Obtain tiling filter applied result images.

Figure 1 explains the concept of the proposed method. As we can see, tiling effect is reflected by four parameters $\mathrm{S}_{\mathrm{HB}}, \mathrm{S}_{\mathrm{VB}}, \mathrm{S}_{\mathrm{HSB}}$, and $\mathrm{S}_{\mathrm{VSB}}$, and their corresponding random function output.

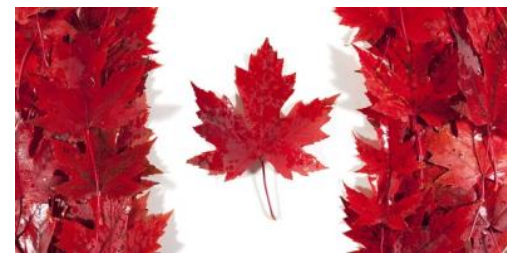

(a)

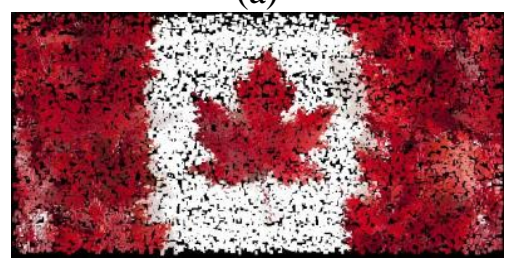

(c)

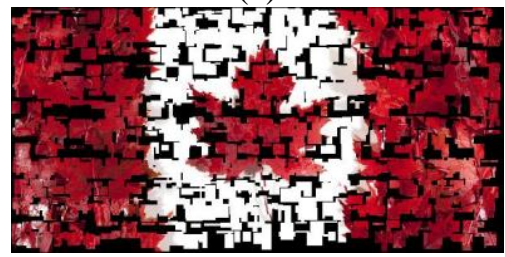

(e)

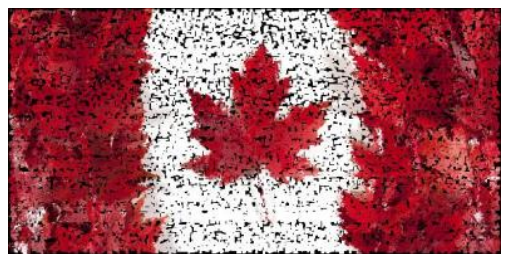

(b)

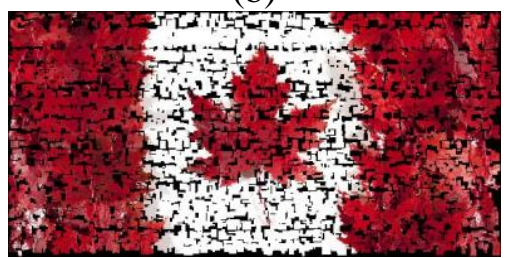

(d)

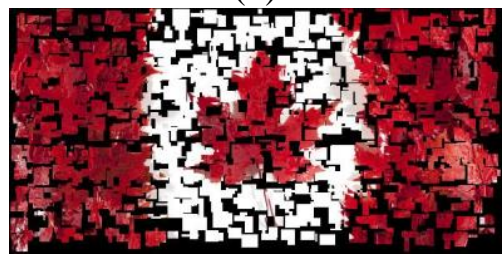

(f)

Figure 2. (a) Original 'Canadian Flag' Image. Tiled Images with (b) Parameter Set (5,5), (c) Parameter Set (5,10), (d) Parameter Set $(10,5)$, (e) Parameter Set $(20,5)$, and (f) Parameter Set $(20,10)$.

\subsection{Definition of the Proposed Method}

Figure 2 shows some effects of original 'Canadian flag' image with different parameter sets. For instance, Figure 2(b) shows implemented image with the parameter set $(5,5)$. In the same manner, Figure 2(b-f) are obtained where parameter sets $(5,10),(10,5),(20,5)$, and (20, 10) were used. The flowchart of the proposed method is drawn in Figure 3.

When $S_{\mathrm{HB}}$ and $S_{\mathrm{VB}}$ (or, $S_{\mathrm{HSB}}$ and $S_{\mathrm{VSB}}$ ) have different values, results images may be shown as Figure 4. Note that Figure 4(a) is result image with parameters $S_{\mathrm{HB}}=5, S_{\mathrm{VB}}=20, S_{\mathrm{HSB}}=10$, and $S_{\mathrm{VSB}}=10$, and Figure 4(b) is result image with parameters $S_{\mathrm{HB}}=20, S_{\mathrm{VB}}=5, S_{\mathrm{HSB}}=10$, and $\mathrm{S}_{\mathrm{VSB}}=10$. 


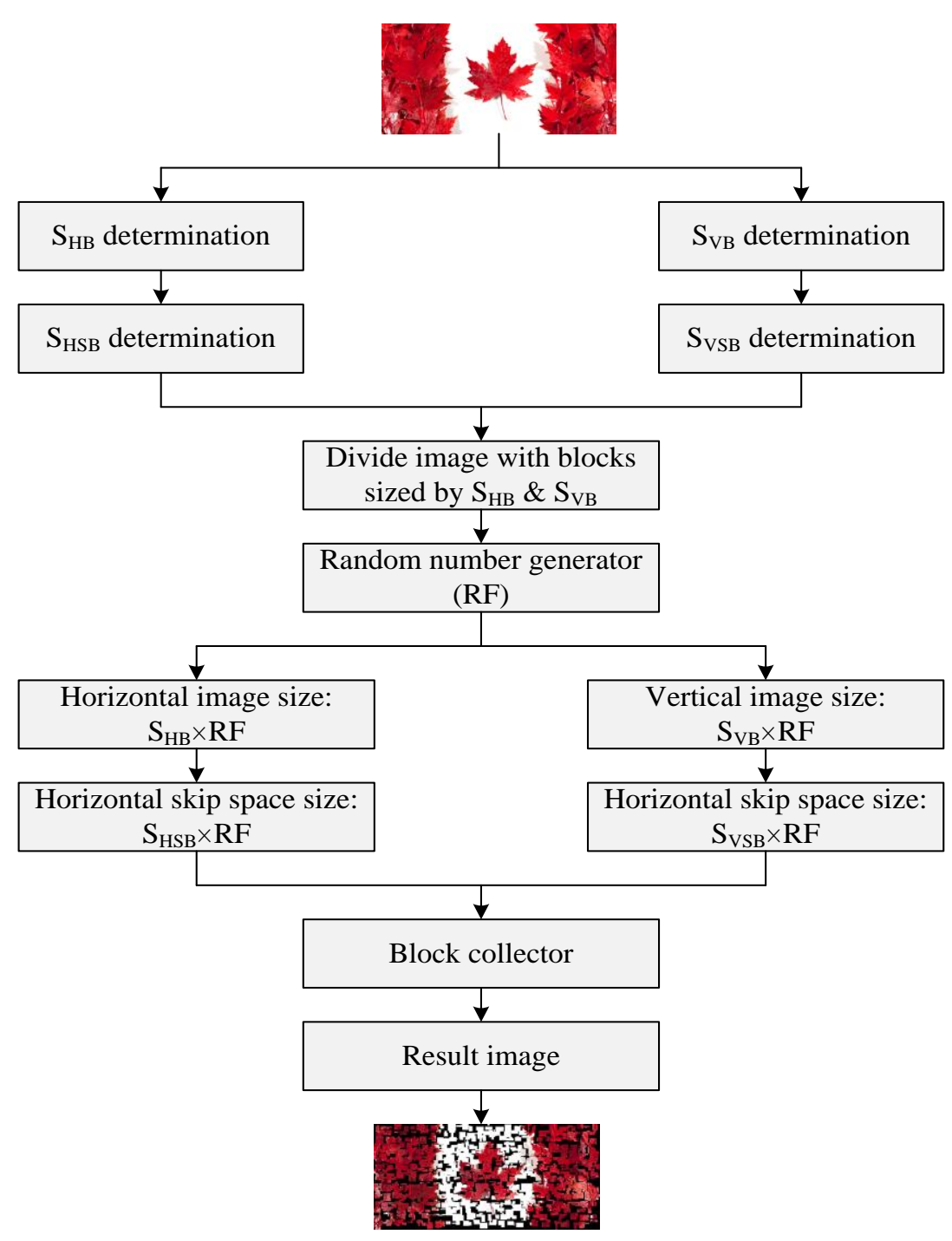

Figure 3. Flowchart of the Proposed Algorithm to Generate Tiling Effect

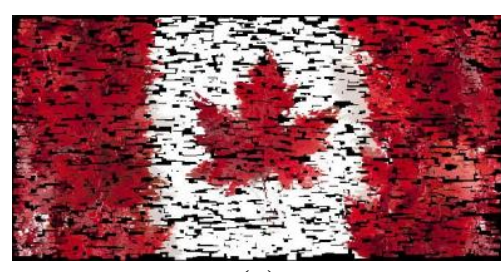

(a)

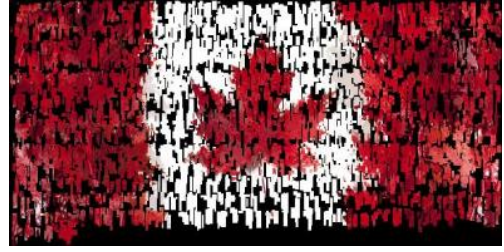

(b)

Figure 4. Result Images with (a) $S_{H B}=5, S_{V B}=20, S_{H S B}=10$, and $S_{V S B}=10$, (b) $S_{H B}=20, S_{V B}=5, S_{H S B}=10$, and $S_{V S B}=10$

\section{Experimental Results}

Our simulation is based on an $18 \mathrm{McM}$ images. The size of all images is $500 \times 500$. The test images are three channel color images. Figure 5 shows $18 \mathrm{McM}$ images. Titling effect was given to original images with different parameters: $\mathrm{S}_{\mathrm{HB}}, \mathrm{S}_{\mathrm{VB}}, \mathrm{S}_{\mathrm{HSB}}$, and $\mathrm{S}_{\mathrm{VSB}}$. 


\subsection{Objective Performance Metrics}

We used three objective metrics: peak signal-to-noise ratio (PSNR), CPSNR, MSE (mean squared error), and FSIM (Feature SIMilarity).

The first metric is PSNR, which is calculated as,

$$
\begin{aligned}
P S N R & =20 \log _{10} M A X-10 \log _{10} M S E, \\
& =10 \log _{10} \frac{M A X^{2}}{M S E},
\end{aligned}
$$

where MAX is 255 .

The second metric is MSE, which is calculated as,

$$
M S E=\frac{1}{w \times h} \sum_{x=0}^{w-1} \sum_{y=0}^{h-1}[\operatorname{ori}(x, y)-\operatorname{rec}(x, y)]^{2},
$$

where $\operatorname{ori}(x, y)$ and $\operatorname{rec}(x, y)$ stand for original and restored pixels at the location of $(x, y)$, and $w$ and $h$ are sizes of monochrome image.

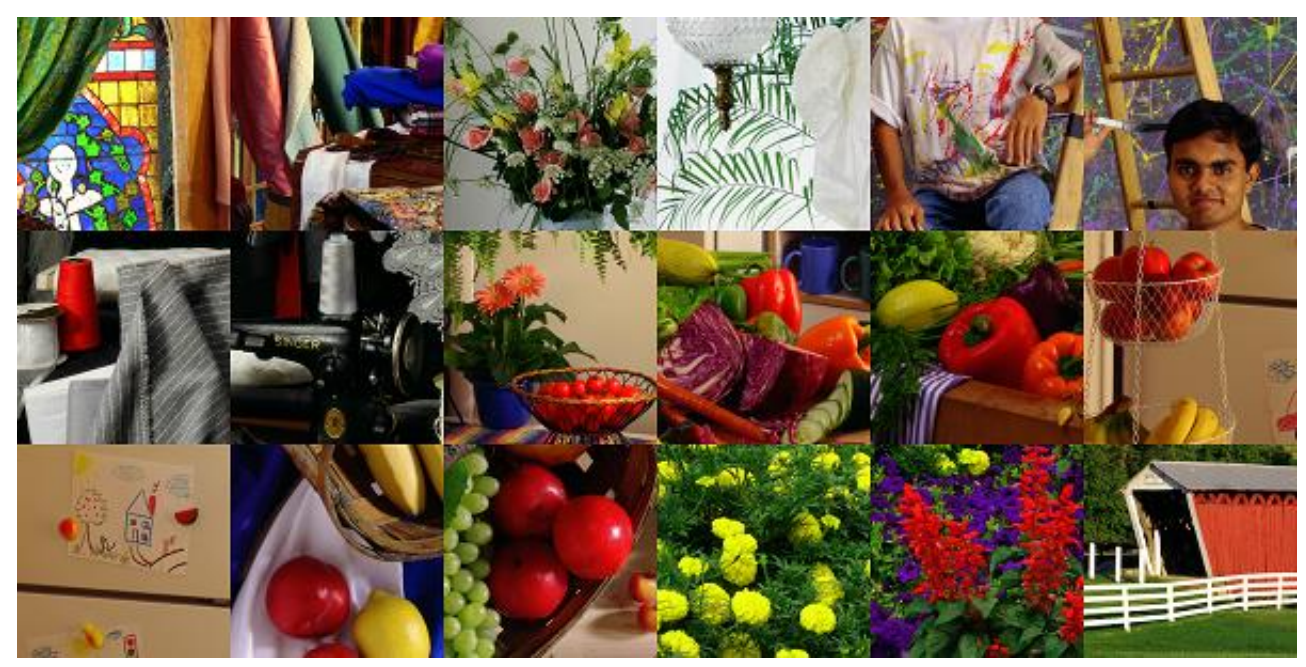

Figure 5. Test Images: McM Dataset

Finally, FSIM is Feature SIMilarity metric, which is calculated as,

$$
F S I M=\frac{\sum_{x \in \Omega} S_{L}(x) \cdot P C_{m}(x)}{\sum_{x \in \Omega} P C_{m}(x)},
$$

where $\Omega$ stands for the whole image, PC is phase congruency, $S_{L}(x)$ is,

$$
S_{L}(x)=\left[S_{P C}(x)\right]\left[S_{G}(x)\right]
$$

\subsection{Objective Performance Comparison}

We have six different scenarios with different parameters. We assumed parameters $S_{\mathrm{HB}}$ and $S_{\mathrm{VB}}$ are identical, and parameters $\mathrm{S}_{\mathrm{HSB}}$ and $\mathrm{S}_{\mathrm{VSB}}$ are the same.

Size parameter sets were considered, they are:

Scenario 1: $\mathrm{S}_{\mathrm{HB}}=5, \mathrm{~S}_{\mathrm{VB}}=5, \mathrm{~S}_{\mathrm{HSB}}=5, \mathrm{~S}_{\mathrm{VSB}}=5$,

Scenario 2: $\mathrm{S}_{\mathrm{HB}}=5, \mathrm{~S}_{\mathrm{VB}}=5, \mathrm{~S}_{\mathrm{HSB}}=10, \mathrm{~S}_{\mathrm{VSB}}=10$, 
Scenario 3: $\quad \mathrm{S}_{\mathrm{HB}}=5, \mathrm{~S}_{\mathrm{VB}}=5, \mathrm{~S}_{\mathrm{HSB}}=15, \mathrm{~S}_{\mathrm{VSB}}=15$,

Scenario 4: $\quad \mathrm{S}_{\mathrm{HB}}=5, \mathrm{~S}_{\mathrm{VB}}=5, \mathrm{~S}_{\mathrm{HSB}}=20, \mathrm{~S}_{\mathrm{VSB}}=20$,

Scenario 5: $\mathrm{S}_{\mathrm{HB}}=10, \mathrm{~S}_{\mathrm{VB}}=10, \mathrm{~S}_{\mathrm{HSB}}=5, \mathrm{~S}_{\mathrm{VSB}}=5$,

Scenario 6: $\quad \mathrm{S}_{\mathrm{HB}}=20, \mathrm{~S}_{\mathrm{VB}}=20, \mathrm{~S}_{\mathrm{HSB}}=5, \mathrm{~S}_{\mathrm{VSB}}=5$.

Figure 6 shows the legend of MSE and PSNR comparisons.

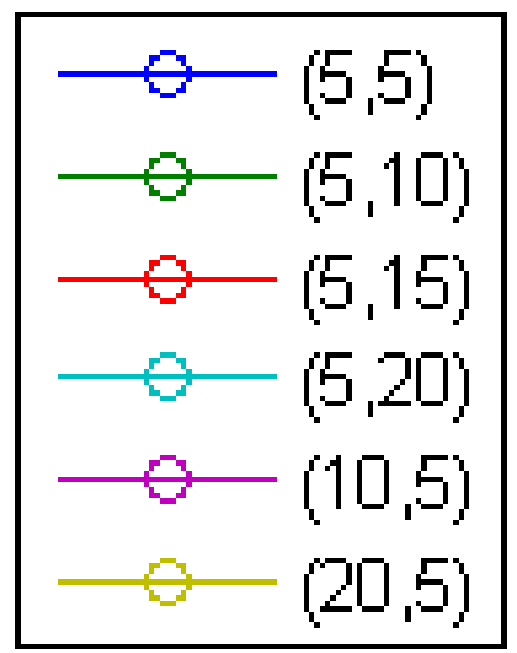

Figure 6. Legend of Objective Performance Comparison

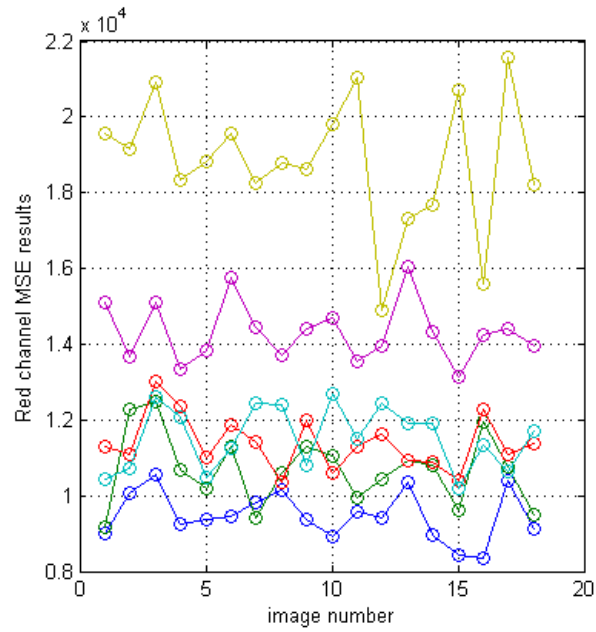

(a)

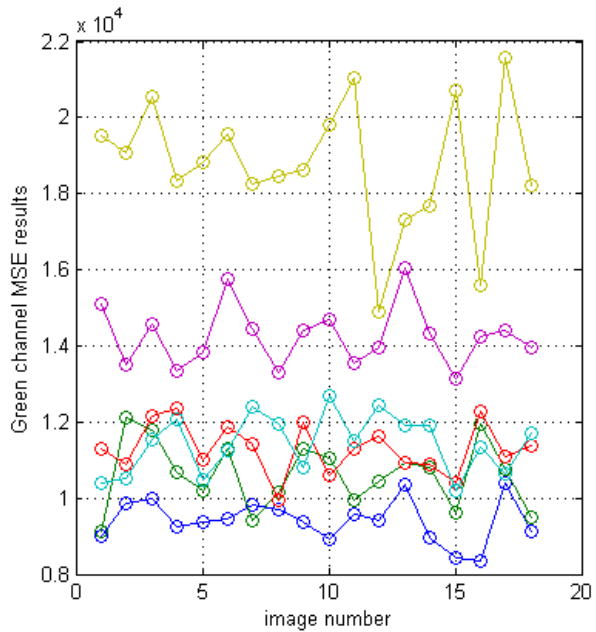

(b) 


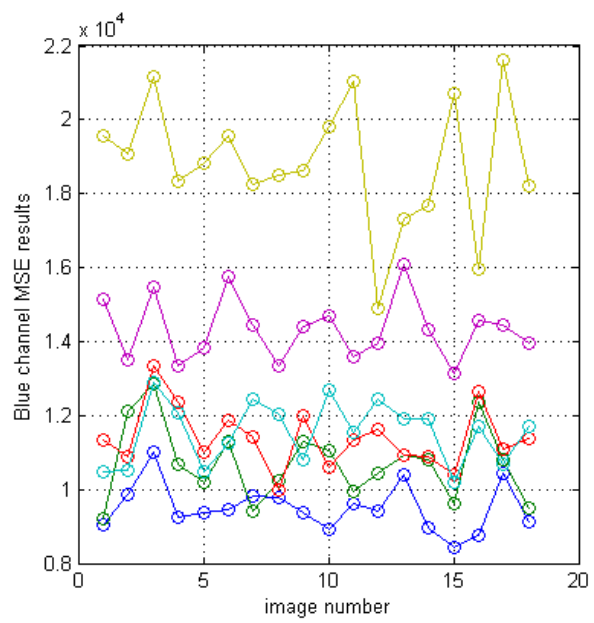

(c)

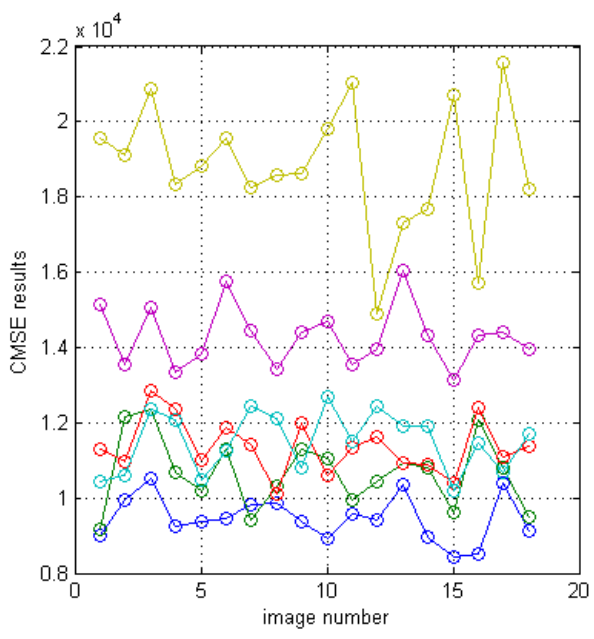

(d)

Figure 7. Performance Comparison using MSE Metric: (a) Red Channel, (b) Blue Channel, (c) Green Channel, and (d) Color MSE

Figures 7 and 8 compare MSE and PSNR results. Table 1 compares FSIM results for six scenarios.

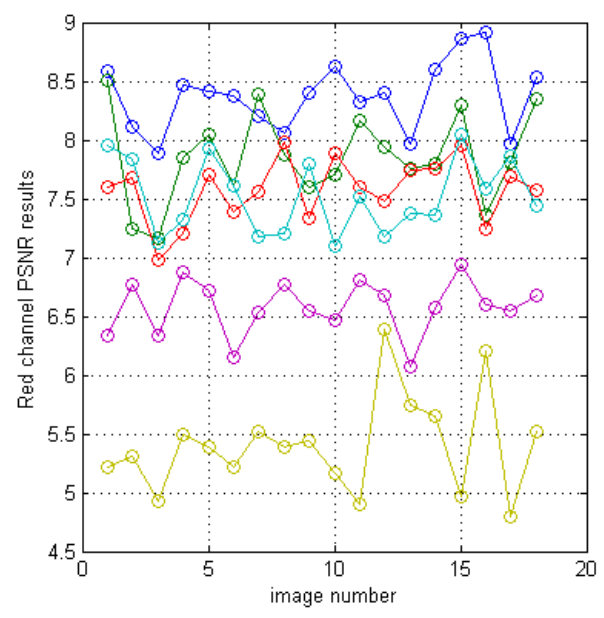

(a)

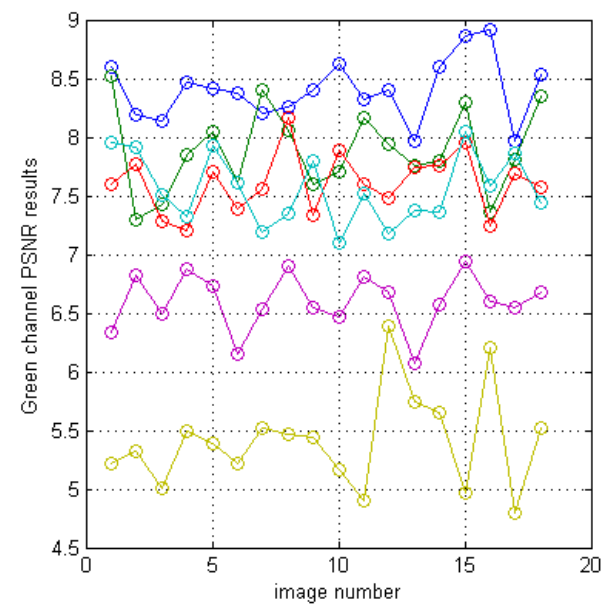

(b) 


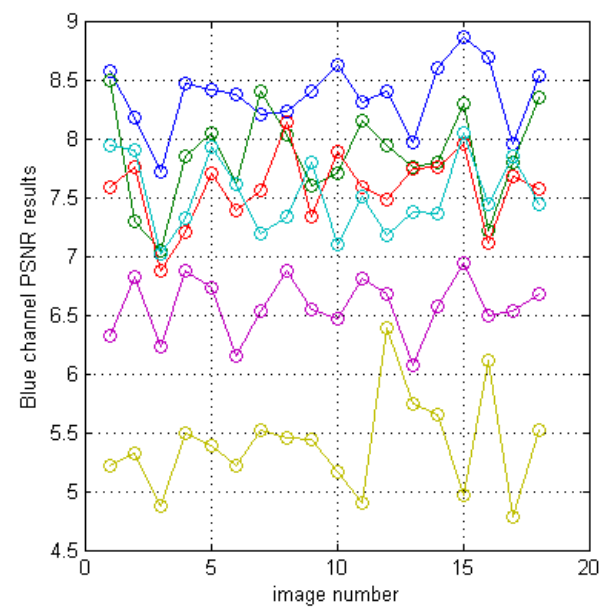

(c)

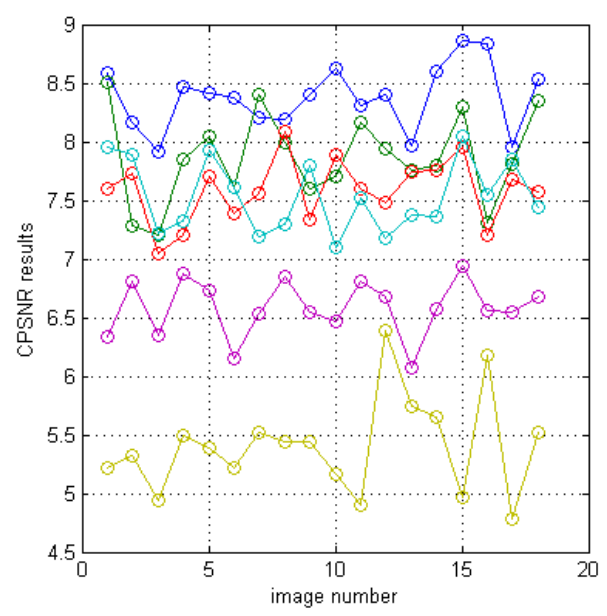

(d)

Figure 8. Performance Comparison using PSNR Metric: (a) Red Channel, (b) Blue Channel, (c) Green Channel, and (d) Color PSNR

Table 1. Shows FSIM Performance Comparison

\begin{tabular}{|c|c|c|c|c|c|c|}
\hline & Scenario 1 & Scenario 2 & Scenario 3 & Scenario 4 & Scenario 5 & Scenario 6 \\
\hline $\begin{array}{c}\text { Average } \\
\text { FSIM }\end{array}$ & 0.2128 & 0.2011 & 0.1959 & 0.2008 & 0.2753 & 0.4291 \\
\hline
\end{tabular}

\subsection{Visual Performance Comparison}

In this subsection, visual performance comparison is provided in Figures 9-11. Figure 9 shows original \#1 and \#2 McM images.

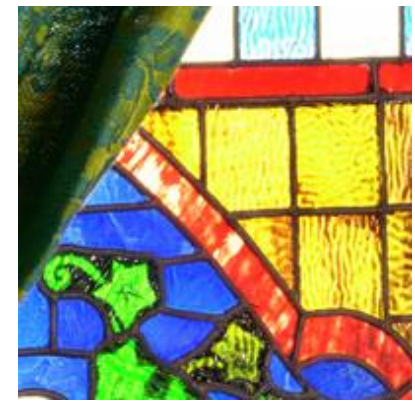

(a)

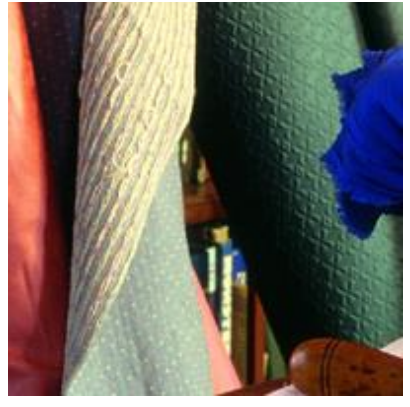

(b)

Figure 9. Original McM Images: (a) \#1 Image, (b) \#2 Image

With different parameter sets, different titling effects were obtained. 


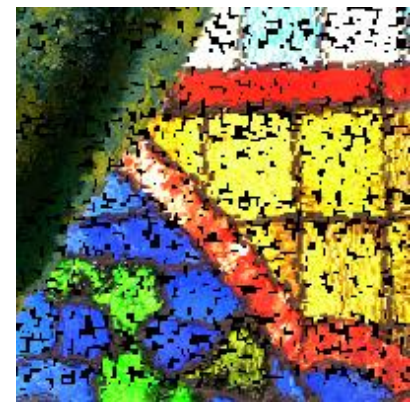

(a)

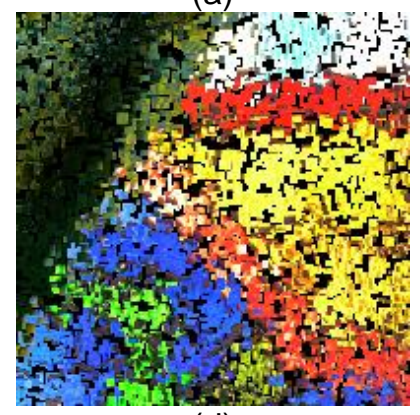

(d)

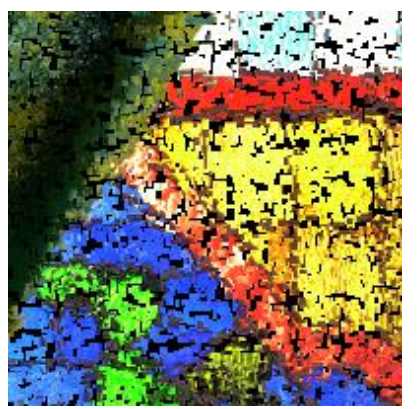

(b)

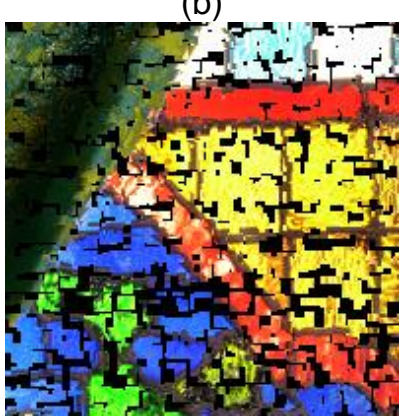

(e)

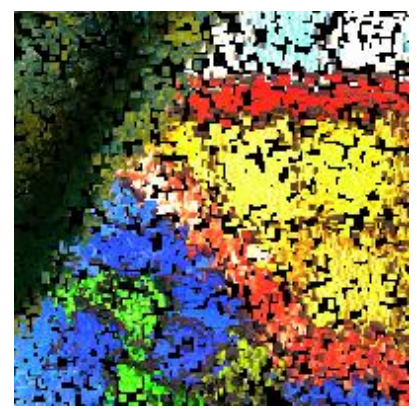

(C)

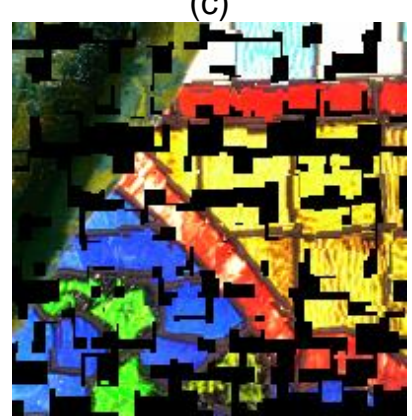

(f)

Figure 10. Results on \#1 McM Image with Different Parameter Sets: (a) Scenario 1, (b) Scenario 2, (c) Scenario 3, (d) Scenario 4, (e) Scenario 5, and (f) Scenario 6

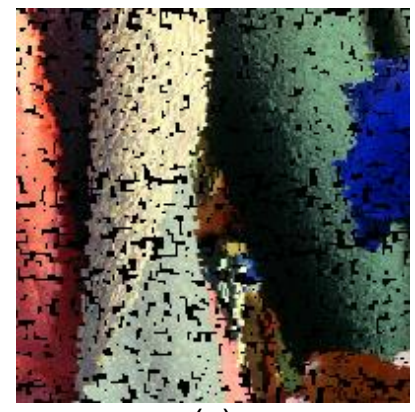

(a)

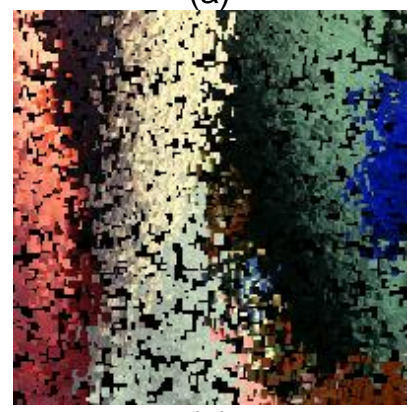

(d)

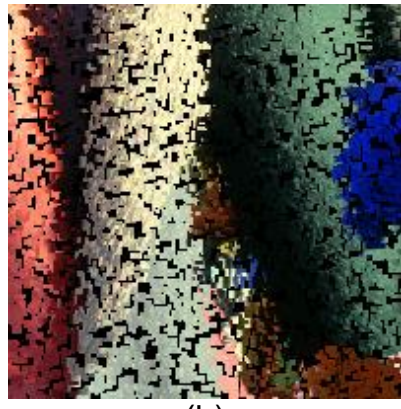

(b)

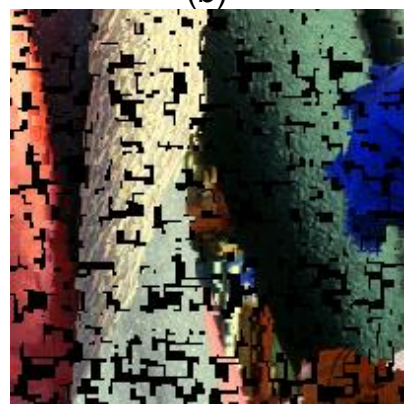

(e)

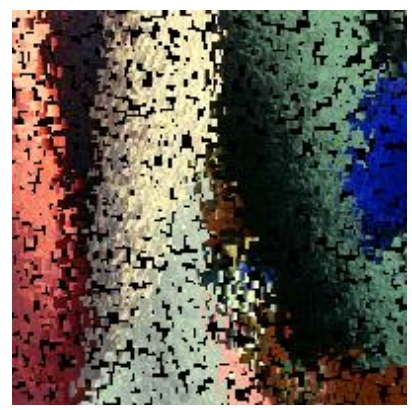

(c)

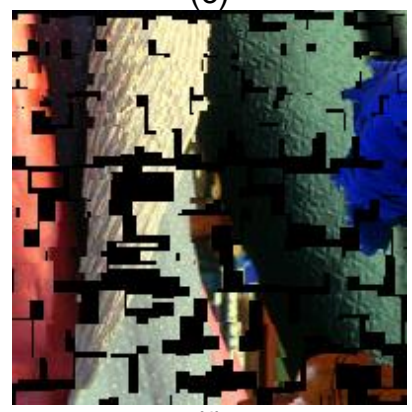

(f)

Figure 11. Results on \#2 McM Image with Different Parameter Sets: (a)

Scenario 1, (b) Scenario 2, (c) Scenario 3, (d) Scenario 4, (e) Scenario 5, and (f) Scenario 6 


\section{Conclusions}

This paper presents a tile effect generating technique. The provided method cuts a provided image into different sized tiles. Four parameters were used to determine the size of horizontal and vertical block, size of skip blocks in horizontal and vertical direction. The provided approach is tested on McM dataset in terms of PSNR, CMSE and FSIM metrics. In addition, visual performance is comparison in detail.

\section{Acknowledgements}

This work was supported by the National Research Foundation of Korea(NRF) Grant funded by the Korean Government(MSIP)(2014025627).

This paper is a revised and expanded version of a paper entitled "Performance Analysis on Tiling Effect," presented at "The 3rd International Conference on Electrics, Electronics, and Computer Science (EEC 2014)," held on October 24-26, 2014 at Liberty Central Saigon Hotel, Hochimin, Vietnam.

\section{References}

[1] N. Ahmed and A. A. Khan, "An efficient image buffering and tiling algorithm for multiple channel image acquisition for space missions," in Proc. ICASE 2013, (2013), pp. 1-5.

[2] S. D. Thepade and S. Erandole, "Effect of tiling in image compression using wavelet transform \& hybrid wavelet transform for cosine \& kekre transforms," in Proc. ICE-CCN 2013, (2013), pp. 753-758.

[3] A. M. Kuruvilla, "Tiled Image Container for Web Compatible Compound Image Compression," in Proc. IEEE SITIS 2012, (2012), pp. 182-187.

[4] M. J. Powell, J. R. Hughes, N. C. Bird, C. Glasse, and T. R. King, "Seamless tiling of amorphous silicon photodiode-TFT arrays for very large area X-ray image sensors," IEEE Trans. Medical Imaging, vol. 17, no. 6, (1998), pp. 1080-1083.

[5] K. Hua, I. Pollak and M. Comer, "Optimal Image Tilings with Application to Video Compression," in Proc. ICIP2006, (2006), pp. 2497-2500.

[6] C. Jin, Q. Deng and J. Liu, "Computer Virus Propagation Model Based on Variable Propagation Rate," IJAST, vol. 1, (2008) December, pp. 29-34.

[7] C. Hsieh, Y. Wu, and K. Hung, "Hybrid Watermarking Scheme for Halftone Images," IJAST, vol. 1, (2008) December, pp. 9-20.

[8] S. Lee, J. Yang, and G. Jeon., "Performance Analysis on Tiling Effect," in Proc. EEC2014, (2012) December.

\section{Author}

Gwanggil Jeon received the BS, MS, and PhD (summa cum laude) degrees in Department of Electronics and Computer Engineering from Hanyang University, Seoul, Korea, in 2003, 2005, and 2008, respectively.

From 2008 to 2009, he was with the Department of Electronics and Computer Engineering, Hanyang University, from 2009 to 2011, he was with the School of Information Technology and Engineering (SITE), University of Ottawa, as a postdoctoral fellow, and from 2011 to 2012, and he was with the Graduate School of Science \& Technology, Niigata University, as an assistant professor. He is currently an assistant professor with the Department of Embedded Systems Engineering, Incheon National University, Incheon, Korea. His research interests fall under the umbrella of image processing, particularly image compression, motion estimation, demosaicking, and image enhancement as well as computational intelligence such as fuzzy and rough sets theories.

He was the recipient of the IEEE Chester Sall Award in 2007 and the 2008 ETRI Journal Paper Award. 